\title{
Experimental results on antiproton-nuclei annihilation cross section at very low energies
}

H. Aghai-Khozani ${ }^{1,2}$, D. Barna ${ }^{3,4}$, M. Corradini ${ }^{5,6}$, R. Hayano ${ }^{4}$, M. Hori ${ }^{1,4}$, T. Kobayashi ${ }^{4}$, M. Leali $^{5,6}$, E. Lodi-Rizzini ${ }^{5,6}$, V. Mascagna ${ }^{5, a}$, M. Prest ${ }^{7,8}$, A. Soter ${ }^{1}$, K. Todoroki ${ }^{4}$, E. Vallazza ${ }^{9}$, L. Venturelli ${ }^{5,6}$, and N. Zurlo $0^{5,6}$

${ }^{1}$ Max-Planck-Institut für Quantenoptik, Hans-Kopfermann-Strasse 1, D-85748 Garching, Germany

${ }^{2}$ Physics Department, CERN, 1211 Geneva 23, Switzerland

${ }^{3}$ Wigner Institute for Particle and Nuclear Physics, H-1525 Budapest, Hungary

${ }^{4}$ Department of Physics, University of Tokyo. Tokyo 113-0033, Japan

${ }^{5}$ Dipartimento di Ingegneria dell'Informazione, Università di Brescia, I-25123 Brescia, Italy

${ }^{6}$ Istituto Nazionale di Fisica Nucleare, Gruppo Collegato di Brescia, I-25123 Brescia, Italy

${ }^{7}$ Università degli Studi dell'Insubria, I-22100 Como, Italy

${ }^{8}$ Istituto Nazionale di Fisica Nucleare, Sezione di Milano Bicocca, I-20126 Milano, Italy

${ }^{9}$ Istituto Nazionale di Fisica Nucleare, Sezione di Trieste, I-34127 Trieste, Italy

\begin{abstract}
Investigating the antiproton cross section on nuclei at low energies $(1 \mathrm{eV}-$ $1 \mathrm{MeV}$ ) is of great interest for fundamental cosmology and nuclear physics as well. The process is of great relevance for the models which try to explain the matter/antimatter asymmetry in the universe assuming the existence of the so-called "island" where antinucleon-nucleon annihilations occur in the border region [1]. For the nuclear physics point of view, the annihilation process is considered a useful tool to evaluate the neutron/proton ratio probing the external region of the nucleus. Moreover, the cross section measured at LEAR in the 80s-90s showed an unexpected behaviour for energies below $1 \mathrm{MeV}$. The results showed a saturation with the atomic mass number against the $\mathrm{A}^{2 / 3}$ trend which is known for higher energies.

The ASACUSA collaboration at CERN measured 5.3 MeV antiproton annihilation cross section on different nuclei whose results demonstrated to be consistent with the blackdisk model with the Coulomb correction [2]. So far, experimental limits prevented the data acquisition for energies below $1 \mathrm{MeV}$. In 2012 the $100 \mathrm{keV}$ region has been investigated for the first time [3].

We present here the results of the experiment.
\end{abstract}

\section{Introduction}

At the present days the only existing source of low energy antiprotons is the AD (Antiproton Decelerator), the successor of LEAR at CERN, which is working since 1999 (see a review article in [4]). A pulsed beam is delivered to the experimental lines with a momentum $100 \mathrm{MeV} / \mathrm{c}$ and the main extraction cycle is about 1 spill/minute, being a single spill a $\sim 200 \mathrm{~ns}$ bunch of about $3 \times 10^{7} \bar{p} \mathrm{~s}$.

\footnotetext{
ae-mail: valerio.mascagna@ing.unibs.it
} 
The ASACUSA collaboration ${ }^{1}$ has a wide physics program including antiprotonic helium spectroscopy, antihydrogen formation and beam production, atomic and nuclear collisions.

This paper is about the results of the $\bar{p}$-nuclei cross section measurements at the AD at two different kinetic energies of the incoming antiprotons (5.3 MeV and $130 \mathrm{keV})$.

\section{Experiment at $5.3 \mathrm{MeV}$}

Antiprotons delivered by the $\mathrm{AD}$ at $5.3 \mathrm{MeV}$ are addressed to a thin target foil in a region surrounded by layers of scintillating fibers which track the charged pions emitted when an antiproton annihilates. This permits the spatial position and time of each annihilation to be measured [5-7].

For each target the annihilation cross section is obtained by counting the annihilations in the target and the ones on the lateral wall due to the antiprotons scattered from the target foil.

With this scheme, the pbar annihilation cross section on medium and heavy nuclei (Mylar, Ni, Sn and Pt) has been measured for the first time in this energy range [2] and the results (see Fig. 1) are in agreement with the black disk model with the contribution of the Coulomb interaction between the antiproton and the nucleus at low energy [8].

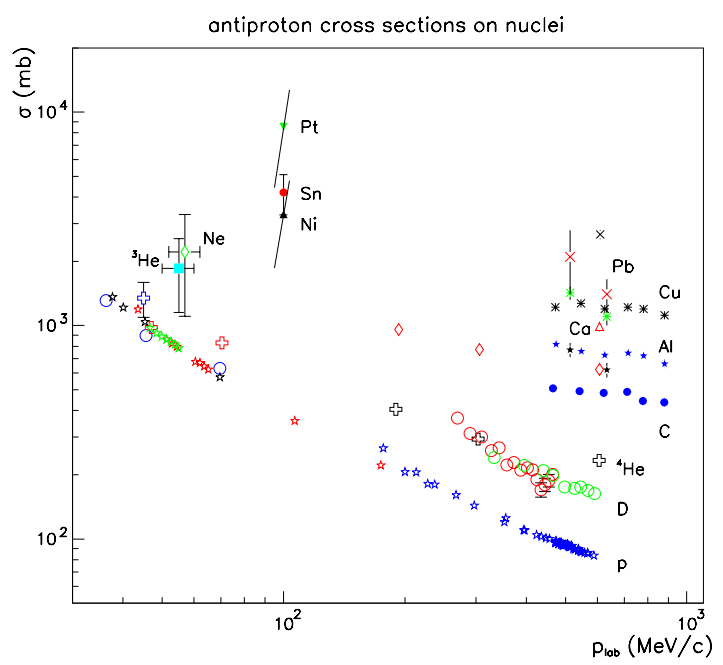

Figure 1. Experimental results for the $5.3 \mathrm{MeV}$ measurements for platinum (green triangle), tin (red circle) and nichel (black triangle) overlapped to existing data (see the fully referenced Figure in [2]).

\section{Down to $130 \mathrm{keV}$}

In 2012 the experiment has been performed using a radio-frequency quadrupole decelerator (RFQD) which reduces the $\bar{p}$ energy to $130 \mathrm{keV}$. A scheme of the setup is shown in Fig. 2: a magnetic spectrometer ("dogleg") transports the decelerated antiprotons toward the target region which is shielded with a wall made of iron $(0.8 \mathrm{~m})$ and concrete $(2 \mathrm{~m})$ and contained in a large cylindrical vessel $(1.7 \mathrm{~m}$ long, $60 \mathrm{~cm}$ diameter). The shielding is needed to reduce the background from stray $\bar{p}$ annihilations.

\footnotetext{
${ }^{1}$ http://asacusa.web.cern.ch
} 
The beam is then focused on the target region by means of an electrostatic quadrupole [9].

A beam profile monitor [10] with a grid of electrode pads fabricated on a normal glass-epoxy circuitboard was placed at the occurrence at the target position for beam tuning.

The charged pions emitted from annihilations are detected by $\sim 600$ plastic scintillator hodoscopes read out by multi-anode PMTs [11] and MPPCs [12] and dedicated frontend electronics. The main information coming out from the data acquisition is the time profile of the hits on the different detectors.

A Cherenkov detector [13] is used as a reference for the beam intensity on a spill-by-spill basis.

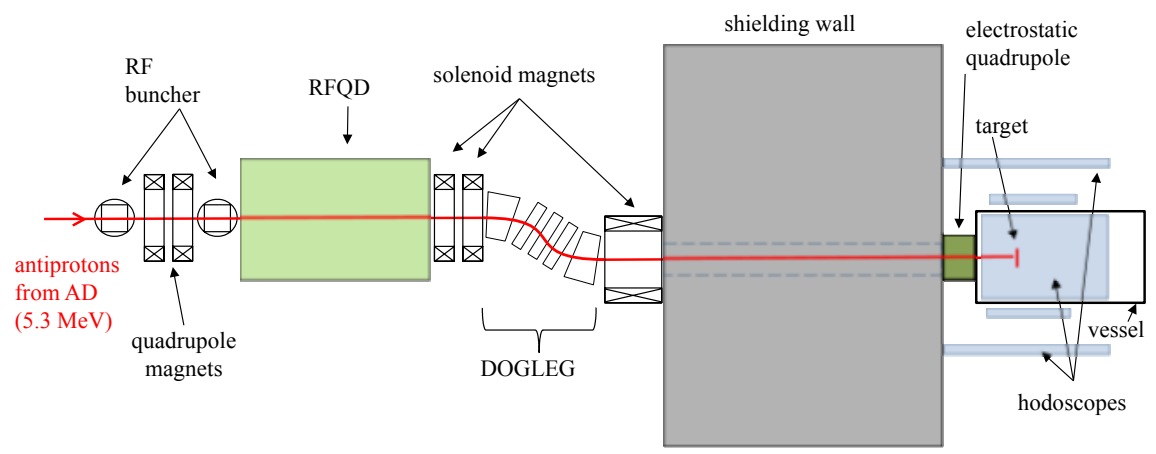

Figure 2. ASACUSA beam line at the CERN AD for the annihilation cross section experiment at $130 \mathrm{keV}$.

The targets have been made at Technische Universität München and consist of different $70 \mathrm{~nm}$ carbon foils with either platinum ( $\sim 5 \mathrm{~nm}$ ) or palladium ( $\sim 19 \mathrm{~nm})$ sputtered on it, or nothing (carbon alone). The annihilation cross section measurements can be performed mainly thanks to the time separation between the annihilations occurring in the target and the other ones on the lateral walls or at the end of the vessel. The validity of this method is shown in Fig. 3, where the time profiles of the 3 targets are shown: the target region can be clearly separated from the annihilation of the whole bunch on the end of the vessel (the huge amount of events at $\sim 1450 \mathrm{~ns}$ ) and from the hits due to the Rutherford-scattered $\bar{p} \mathrm{~s}$ in the target as well.

\section{Conclusions}

The annihilation cross section of antiprotons on different nuclei has been measured at low energies. At $5.3 \mathrm{MeV}$ the results are in agreement with a black disk model with a Coulomb correction, at $130 \mathrm{keV}$ 


\section{EPJ Web of Conferences}

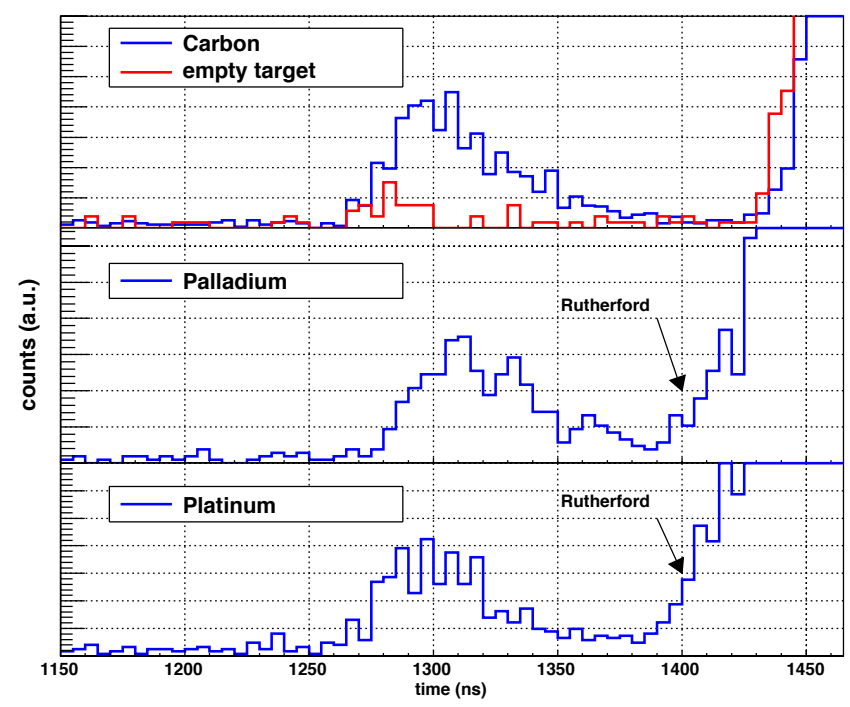

Figure 3. Time profile of the $\bar{p}$-annihilations as seen by one of the detectors. The target region in the 3 cases (carbon only, palladium and platinum) can be seen at $\sim 1300 \mathrm{~ns}$.

the feasability of the experiment has been demostrated by clearly observing $\bar{p}$-nucleus annihilations in the very low energy range of about $100 \mathrm{keV}$ for the first time. The analysis of these events is under process.

\section{References}

[1] A. G. Cohen, A. de Rujúla and S. L. Glashow, The Astrophys. Journ. 495 (1998), 539.

[2] A. Bianconi et al., Phys. Lett. B 704, (2011) 461.

[3] H. Aghai-Khozani et al., Eur. Phys. J. Plus (2012) 127: 125.

[4] M. Hori and J. Waltz, Progress in Particle and Nuclear Physics, Volume 72, September 2013, Pages 206-253.

[5] M. Corradini et al., Nucl. Instr. and Meth in Phys. Res. A 711 (2013), 12-20.

[6] M. Corradini et al., Hyperfine Interact (2012) 213:31-39.

[7] V. Mascagna et al., Nuclear Physics B (Proc. Suppl.) 172 (2007) 299-302.

[8] C. J. Batty, E. Friedman, and A. Gal, Nucl. Phys. A 689, 721 (2001).

[9] M. Hori et al., Phys. Rev. Lett. 91, 123401 (2003).

[10] K. Todoroki and M. Hori, JINST 7, C02052 (2012).

[11] M. Corradini et al., in preparation.

[12] A. Soter et al., in preparation.

[13] M. Hori et al., Nucl. Instr. and Methods A 496, 102 (2003). 